- verstärkt Partizipationsmöglichkeiten am gesellschaftlichen, politischen und kulturellen Leben zu schaffen, auch durch Prozesse der interkulturellen Öffnung;

- Migrantinnen noch stärker in den Dialog über Erziehungsund Bildungsziele, Werte und Grundlagen unserer Gesellschaft einzubinden;

- klar zu machen, von welch zentraler Bedeutung das Thema Bildung und das Erlernen der deutschen Sprache für ihre Zukunft und die Zukunft ihrer Kinder ist.

Im Hinblick auf diese Zielrichtungen war es übrigens ein wichtiger Schritt, dass seit dem Inkrafttreten des Zuwanderungsgesetzes am 1. Januar 2005 im Rahmen des Integrationskurssystems spezielle Frauenkurse, zum Teil auch vorbereitende Seminarmaßnahmen, durchgeführt werden. Außerdem wurden entsprechend der Zielbestimmung des Nationalen Integrationsplans zum 1. Januar 2008 als weitere Themenschwerpunkte der Frauenkurse die Sprachentwicklung und der Spracherwerb der Kinder durch die Eltern sowie der Schutz vor häuslicher Gewalt aufgenommen.
Lassen Sie mich noch ein Thema ansprechen, mit dem sich die baden-württembergische Landesregierung seit Jahren intensiv beschäftigt: die Zwangsverheiratung von Mädchen und jungen Frauen. Es wird eine wichtige Aufgabe der neuen Bundesregierung sein, die Zwangsheirat als umfassenden Straftatbestand in das Strafgesetzbuch aufzunehmen, die zivilrechtliche Rechtsstellung von Opfern von Zwangsverheiratungen zu verbessern und im Aufenthaltsrecht ein verbessertes Rückkehrrecht in Fällen der Heiratsverschleppung zu verankern.

Meine sehr geehrten Damen und Herren: Das Potenzial von Frauen mit Migrationshintergrund liegt - trotz vieler positiver Entwicklungen - oft noch brach. Es gilt, die Schlüsselfunktion dieser Frauen verstärkt zu nutzen und Teilhabemöglichkeiten zu schaffen, um den Integrationsprozess weiter voranzubringen. In diesem Sinne wünsche ich Ihnen eine interessante und erfolgreiche Tagung und hoffe, dass Sie auch Gelegenheit finden, einen Eindruck von der Stadt Karlsruhe mit ihren Sehenswürdigkeiten und der typischen badischen Gastlichkeit zu bekommen!

Vielen Dank!

\title{
Integration durch Verfassung Eröffnungsvortrag
}

\section{Prof. Dr. Gertrude Lübbe-Wolff, Richterin des Bundesverfassungs- gerichts, Karlsruhe}

Welche Bedeutung hat die Verfassung für die Integration einer Gesellschaft oder politischen Gemeinschaft?

Die Frage, wie die Integration menschlicher Gesellschaften eigentlich funktioniert, wird gern gleichgesetzt mit der Frage: Was hält eine Gesellschaft zusammen? Diese Frage führt auf eine Merkwürdigkeit vieler Integrationsüberlegungen. Sie scheint vorauszusetzen, dass es ein Etwas gibt, das menschliche Gesellschaften zusammenhält, und schickt den Fragenden auf die Suche nach diesem Etwas. Vor einem Staat oder einer anderen Organisationsform menschlicher Gesellschaft steht man mit dieser Frage wie vor einer Sandkugel: Warum zerbröckelt sie nicht, warum zerrieselt sie nicht in lauter einzelne Sandkörner? Da muss doch irgendetwas für die Kohäsion Verantwortliches beigemischt sein! Dieses Etwas muss man finden, dann hat man die Erklärung für die Kugel, die man vor sich sieht, und damit auch das Backrezept für schöne, feste Sandkugeln.

\section{Die freiheitliche Verfassung - integrationsschwach?}

Die beliebteste Antwort auf die Frage, woraus er denn besteht, der Kitt, der die Gesellschaft zusammenhält, lautet, dass der Zusammenhalt nur durch irgendeine Art von Ähnlichkeit oder spezifischer Gemeinsamkeit, kurz: durch Homogenität, vermittelt und gesichert werden kann. Unterschiedlich sind dann aber wieder die Vorstellungen über die Art des benötigten Homogenitätskitts.

Über Homogenitätslehren, die auf Gleichheit der Rasse oder Ethnie setzten, ist hier kein Wort zu verlieren. Wo in der Staatslehre heute Homogenität als Voraussetzung gelingender politischer Gemeinschaft postuliert wird, kommt die Gemeinsamkeit der „Abstammung“, wenn überhaupt, dann nur noch als ein möglicher homogenitätsstiftender Faktor neben anderen vor, oder man zieht sich ganz auf eine irgendwo im Kulturellen (Gemeinsamkeit der Sprache, der Religion, der Geschichte usw.) angesiedelte Homogenität zurück. Oder, das ist die letzte Schwundstufe, auf irgendeine Form von Überzeugungs- oder Gefühlseinheit. Als der unverzichtbare Homogenitätskitt gilt dann beispielsweise ein Grundkonsens oder ein Wir-Gefühl. Auch die Idee vom Verfassungspatriotismus als einigendem Band gehört hierher.

Homogenitätslehren, die auf einer Art Gesinnungs- oder Gefühlseinheit beharren, haben eine gewisse Tendenz, in Resignation vor den widrigen Weltläuften zu enden: Man bemerkt das Faktum zunehmender gesellschaftlicher Differenzierung, also zunehmender Inhomogenität, erkennt womöglich auch noch, dass eine freiheitliche Verfassung gesellschaftlichen Differenzierungen und der damit einhergehenden Inhomogenität nicht entgegenwirkt, sondern sie im Gegenteil gerade ermöglicht und schützt, und sieht deshalb das haltende Gemeinsame der Erosion preisgegeben. 
Der freiheitliche Verfassungsstaat, so habe ich es erst kürzlich wieder bei einer Konferenz gehört, trägt die Tendenz zur Selbstdestruktion in sich, weil die Differenzen, die er freisetzt, die Gemeinsamkeit verzehren, die zu seiner Erhaltung nötig wäre.

Auch in der berühmten Böckenfördeschen Feststellung, der freiheitliche, säkulare Staat lebe von Voraussetzungen, die er nicht selbst garantieren könne, schimmert ein Element solcher Sorge durch.

Diese Feststellung bringt ja nicht die Banalität zum Ausdruck, dass der Mensch den Erfolg seiner Projekte, und so auch den Fortbestand seines Gemeinwesens, nicht in der Hand hat, sondern sie zielt auf eine Besonderheit des freiheitlichen Verfassungsstaates - auf die Besonderheit, die darin liegt, dass der freiheitliche Verfassungsstaat, als freiheitlicher, seinen Bürgern die Überzeugungen, von denen er lebt, schon von Verfassungs wegen nicht einfach abverlangen kann. Diese Feststellung ist richtig, und sie ist nicht trivial. Gibt sie aber Anlass zur Sorge? Folgt aus ihr, dass der gesellschaftliche Zusammenhalt, die Integration, gerade im freiheitlichen Verfassungsstaat eine besonders prekäre Größe ist?

Nein. Das Problem, das hier gesehen wird, geht nicht aus der Natur des freiheitlichen Verfassungsstaates hervor, sondern aus der Sandkugeltheorie, die die Integration politischer Gemeinschaften nur durch ein zusammenhaltendes Etwas erklären zu können glaubt und zu diesem Etwas die Homogenität ausersehen hat.

\section{Homogenitätstheorien gehen an der entscheidenden Frage vorbei}

Von den unendlich vielen konkreten Fragen, die sich in Bezug auf historische und aktuelle Probleme der Integration und Desintegration politischer Einheiten stellen, muss man sich nur irgendeine beliebige kleine Auswahl vor Augen führen, um zu sehen, dass wir es mit einer Komplexität der Phänomene und Ursachen zu tun haben, vor der solche einfachen Erklärungen mit umfassendem Anspruch sich nur als entweder falsch oder trivial erweisen können. Wie konnten die Mongolen auch nur zeitweise fast das ganze Asien und halb Europa unter ihrer Herrschaft vereinen? Warum ist der habsburgische Vielvölkerstaat zerfallen? Warum funktioniert die Schweiz? Was hat den Holocaust möglich gemacht? Können Hutu und Tutsi in Ruanda versöhnt werden? Woran hängt der Friede zwischen Hindus und Muslimen in Indien, zwischen Christen und Muslimen im Libanon, zwischen Katholiken und Protestanten in Irland? Lässt sich in Afghanistan ein landesweit funktionsfähiger Staat aufbauen, und wenn ja, wie? Häuft die Finanzkrise sozialen Zündstoff an? Fördern Kopftuchverbote für Lehrerinnen die Integration oder erschweren sie sie?

Lauter Integrationsfragen, die ahnen lassen: Die überwölbende, alle diese und Millionen anderer Integrationsfragen einschließende Frage, was politische Gemeinschaften oder was „die Gesellschaft“ zusammenhält oder auseinanderfallen lässt, ist zu groß gestellt, um mit Hinweis auf irgendein einzelnes Etwas unbanal beantwortbar zu sein.

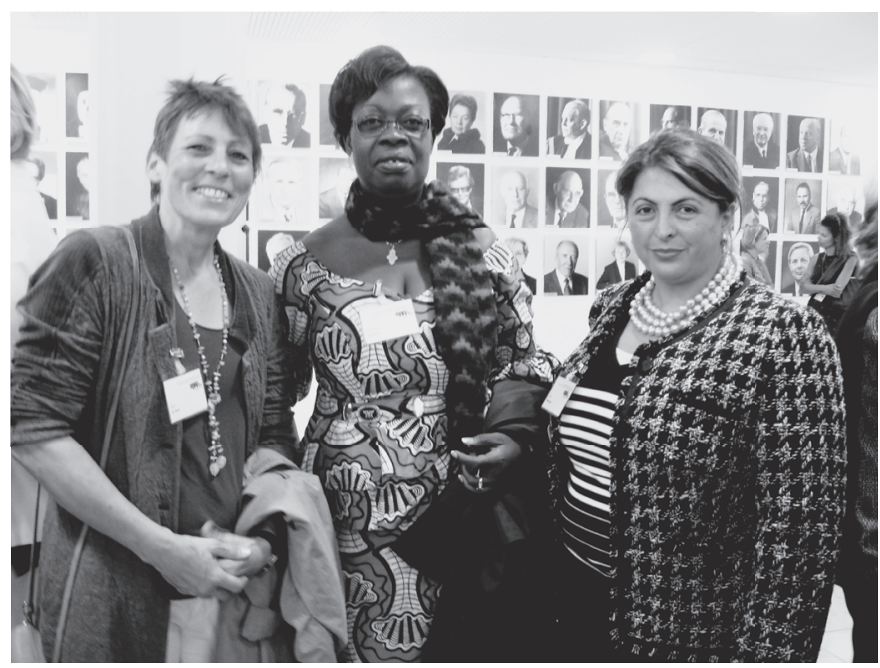

- Afiwa Kafui Kuwonu, Secrétaire Générale Groupe de réflexion et d'action de Femme, Démocratie et Développement (GF2D), Wiss. Mit. Dr. Katja Rodi (links) und Projektmanagerin Nimet Güller-Kaya (rechts) (Foto: RM).

Homogenitätstheorien und Homogenitätspostulate liefern hier nur den Anschein einer Antwort, denn sie gehen vorbei an der unendlichen Komplexität der Erscheinungsformen von Inhomogenität, an der Vielfalt der konkreten Möglichkeiten, aber auch der möglichen Hindernisse erfolgreicher Bewältigung von Inhomogenität. Sie gehen vorbei an der Vielfalt der Gründe, aus denen politische Gemeinschaften als solche Stabilität erlangen oder scheitern können. Und damit auch vorbei an der entscheidenden Frage, wie politische Integration angesichts gegebener Inhomogenitäten möglich ist.

Das ist nun genau die Frage, auf die moderne Verfassungen $\mathrm{zu}$ antworten suchen.

\section{Abgeschaffte Integrationsmittel}

Betrachten wir die moderne Verfassung in ihrer jüngsten Form, als demokratische, freiheitliche Verfassung, dann stellen wir allerdings fest, dass dem so verfassten Gemeinwesen bestimmte Mittel nicht mehr zur Verfügung stehen, die ältere Gemeinwesen zur Stabilisierung ihrer inneren Einheit angewandt haben und gegenwärtige, die nicht freiheitlich-demokratisch verfasst sind, zum Teil noch immer anwenden.

Römische Kaiser verfolgten die Christen. Später, als sie selbst Christen geworden waren, erklärten sie den Abfall vom Christentum zum Verbrechen. Dem italienischen Fürsten des sechzehnten Jahrhunderts konnte Macchiavelli den Rat geben, die Integration hinzueroberter Fürstentümer durch sorgfältige Auslöschung der gesamten bisherigen Herrscherfamilie zu sichern. Im frühen zwanzigsten Jahrhundert betrachtete man die Zwangsumsiedlung als probates Integrationsmittel. Bevölkerungsteile, die als Störung oder Bedrohung der nationalen Einheit galten, wurden zwangsweise dorthin transferiert, wo sie homogenitätshalber hinzugehören schienen. Der 1923 im Vertrag von Lausanne vorgesehene Bevölkerungsaustausch zwischen Griechenland und der Türkei ist das bekannteste Beispiel.

Alle diese und viele andere Instrumente der Einheitssicherung stehen dem freiheitlichen Verfassungsstaat nicht zur Ver- 
fügung. Verfolgung, Vertreibung, Zwangsumsiedlung - all das ist heute auch vom Völkerrecht geächtet und gilt nicht mehr als mögliches Mittel, sondern als Inbegriff des Scheiterns von Integration. Die freiheitliche Verfassung zwingt selbst ihre erklärten Gegner weder zur Bekehrung noch entzieht sie ihnen prinzipiell das Wort.

Was setzt sie aber an die Stelle der Integrationsmittel, die sie abgeschafft hat?

\section{Bewusstseinspflege als Integrationsmittel}

In der deutschen staatsrechtlichen und verfassungstheoretischen Literatur wird die Integrationsfunktion der Verfassung heute meist in ihrer Bedeutung als mögliche Grundlage einer spirituellen Homogenität ins Auge gefasst: als Gegenstand von Einheitsbewusstsein, Wir-Gefühl, Patriotismus.

Die Verfassung kommt dabei vor allem als Ausdruck gemeinsamer Werte und Überzeugungen in den Blick, die im öffentlichen Bewusstsein gerade über die Identifikation mit der Verfassung befestigt und zur Grundlage eines Gemeinschaftsgefühls gemacht werden sollen. Die Aufmerksamkeit konzentriert sich infolgedessen regelmäßig auf Symbolisches: Einheitsrepräsentation durch das Staatsoberhaupt, Flagge, Nationalhymne, Nationalfeiertag, Gestaltung von Einbürgerungsfeiern und so fort.

Das ist auch alles nicht unwichtig, aber nicht das primär Wichtige. Die Ausblendung des Wichtigeren hat in Deutschland Tradition. Schon Rudolf Smend, der deutsche Klassiker zum Thema Integration, hat sich bei diesem Thema vor allem für das interessiert, was den Bürgern eines Staates auf direkte Weise das Gefühl, Bewusstsein oder Erlebnis ihrer Einheit im Staate verschafft. Er unterschied zwischen persönlicher, funktioneller und sachlicher Integration, d.h. der Integration „durch Personen“, durch „Funktionen oder Verfahrensweisen“ und durch „Sachgehalte“. Bei der persönlichen Integration dachte er vor allem an die Repräsentation der Einheit des Volkes und der das Volk vereinenden Gemeinschaftswerte in der Person des Staatsoberhaupts, bei der funktionellen an „kollektivierende Lebensformen“, „Vorgänge, ... die irgendeinen geistigen Gehalt gemeinsam machen oder das Erlebnis der Gemeinschaft verstärken wollen“ - vom „akustische(n) oder motorische(n) Rhythmus gemeinsamer Tätigkeit“ bis zum parlamentarischen Prozess, der in dem „miterlebenden Staatsvolk Gruppenbildung, Zusammenschluss, Bildung einer politischen Gesamthaltung herbeiführt“ (darauf, ob das Parlament gute Beschlüsse oder ob es überhaupt Beschlüsse fasst, komme es insofern gar nicht an), und bei der sachlichen an den Staat als „Sinnverwirklichung“ und „Werttotalität“, durch die allein er als „dauernder einheitlich motivierender Erlebniszusammenhang “ herrschen könne und die deshalb in politischen Symbolen wie Fahnen, Wappen, nationalen Festen, dem Staatsoberhaupt usw. symbolisch repräsentiert werden müssen.

Dass die symbolische Darstellung des Gemeinsamen, das Insbildsetzen des Zustimmungsfähigen einer politischen Ordnung, als Integrationsfaktor wirken kann, wird niemand be- streiten. Ein demokratisches Gemeinwesen tut gut daran, diese Art der verbindenden Kommunikation nicht den Diktatoren und den Demagogen zu überlassen. Dass solche Kommunikation sich mehr ans Gefühl als an die Vernunft wendet und dass sie missbraucht werden kann, ist kein Grund, sie mit prinzipieller Skepsis zu betrachten. Wenn es einer Nation gelingt, positives Gefühl auf etwas so Vernünftiges wie ihre freiheitliche und demokratische Verfassung zu wenden und dieses Vernünftige zu zelebrieren, wie uns das mit der Feier zum sechzigsten Jahrestag des Grundgesetzes am 23. Mai dieses Jahres in Berlin gelungen ist - was könnte erfreulicher sein? In anderen Ländern, wo man das im Fernsehen verfolgen konnte, beneidet man uns darum.

\section{Verfassung als Integrationsprogramm}

Es wäre aber absurd, anzunehmen, dass die integrative Wirkung einer Verfassung vor allem von solchen bewusstseinspflegerischen Aktivitäten, von Symbolisierungen und Zelebrationen abhängt, als käme es auf das, was da symbolisiert wird, erst in zweiter Linie oder überhaupt nicht an. Es kommt darauf an. Denn Menschen sind in der Lage, zwischen den Zeichen und der Realität, für die sie stehen, zu unterscheiden. Jedes Symbol nimmt deshalb über kurz oder lang die Farbe, die Gefühlswerte des Symbolisierten an - wird grau, wenn das Symbolisierte grau ist, wird schal, wenn das Symbolisierte nicht begeistern kann, und stößt ab, wenn das Symbolisierte Widerwillen erregt.

Für das Verständnis der integrativen Funktion von Verfassungen ist darum eine Perspektive aufschlussreicher, die nicht die symbolischen, sondern die operativen Gehalte, die konkreten institutionellen Ordnungsleistungen einer Verfassung in den Blick nimmt.

Moderne Verfassungen erscheinen dann in ihren charakteristischen Grundzügen als Antwort auf die Frage, wie entscheidungsfähige politische Einheit gerade unter den Bedingungen gegebener Inhomogenität ermöglicht werden kann. In dieser Perspektive wird deutlich, dass eine der wesentlichen Integrationsfunktionen moderner Verfassungen gerade in der Lizenz zur Inhomogenität liegt. Moderne Verfassungen sind Integrationsprogramme für differenzierte Gesellschaften.

\section{Integration durch Freiheit}

Besonders augenfällig zeigt sich das in der Geschichte des Urgrundrechts, der Religionsfreiheit.

Die Religionsfreiheit verdanken wir nicht der Suche nach sympathischen Verfassungsinhalten, an die sich ein Wir-Gefühl knüpfen lässt, sondern einer Geschichte der Pluralisierung religiöser Überzeugungen, in der die Zulassung solcher Inhomogenität sich als Voraussetzung friedlicher Koexistenz erwiesen hat. Am Anfang dieser Geschichte standen Religionskriege, aus denen es keinen anderen Ausweg gab als die Anerkennung eines Rechts auf religiöse Differenz. Die Vorstellung, dass funktionsfähige politische Einheit nur auf religiöser Einheit gründen kann, wurde dabei nur schrittweise aufgegeben. 
Die mittelalterliche Lehre von der concordia (Eintracht) als notwendiger Grundlage des Gemeinwesens hat man im sechzehnten und siebzehnten Jahrhundert in der Variante aufrechtzuerhalten gesucht, dass die nötige concordia in dieser oder jener einheitsstiftenden Religion wurzeln könnten, aber doch jeweils nur in einer einzigen. Der Grundsatz „cuius regio, eius religio“" (wessen Gebiet, dessen Religion) des Augsburger Religionsfriedens war Ausdruck dieser Überzeugung. In der Entwicklung der Religionsfreiheit bis hin zu der Bedeutung, in der sie heute Gemeingut moderner Verfassungen ist, zeigt sich das Prinzip der Integration differenzierter Gesellschaften: Die differenzierte Gesellschaft integriert sich nicht durch Homogenitätsansprüche, sondern durch Freiheit, also gerade durch Freilassung und Freisetzung von Unterschieden, und durch die Anerkennung Unterschiedlicher als gleichberechtigt. Der Freiheit können und müssen Grenzen gezogen werden, aber diese Grenzen werden nicht durch Homogenitätsansprüche gezogen oder begründet.

Integration durch gleiche Freiheit heißt: Integration primär durch Freiwilligkeit statt primär durch Zwang, primär durch Zustimmungsfähigkeit statt primär durch Repression, primär durch die Loyalität, die dem Gemeinwesen dadurch zuwächst, dass es seinen Mitgliedern ermöglicht, ihre Ziele zu verfolgen und nach ibren Überzeugungen zu leben, statt durch Zuweisung von Zwangsrollen im gesellschaftlichen Organismus.

Hegel ist der erste, der in dem modernen Prinzip der Freiheit gerade das integrative Potential klar gesehen hat: „Das Prinzip der modernen Staaten hat diese ungeheure Stärke und Tiefe, das Prinzip der Subjektivität sich zum selbständigen Extreme der persönlichen Besonderheit vollenden zu lassen und zugleich es in die substantielle Einheit zurückzuführen und so in ihm selbst diese zu erhalten." (RPh $\$ 260)$.

Das Bundesverfassungsgericht hat zu Recht in seinem Hochschulurteil (1972) von der „allgemeinen fundamentalen Bedeutung der Grundrechte für die Integration einer Demokratie freier, mitverantwortlicher Bürger“ gesprochen.

\section{Integration durch wirtschaftliche Grundrechte und Sozial- staatsprinzip - die Bedeutung der Aufstiegsmobilität}

In dem Integrationsmodell, das auf Einheitsbildung auf der Grundlage gleicher Freiheit setzt, spielt auch das Eigentumsrecht mit den zugehörigen wirtschaftsbezogenen Freiheiten eine zentrale Rolle, soweit es durch gesicherte Privatnützigkeit gesellschaftlichen Wohlstandsnutzen stiftet.

Der Zusammenhang von privatem und gesellschaftlichem Nutzen, von dem abhängt, ob die Eigentumsordnung integrative oder desintegrative Wirkungen entfaltet, ergibt sich allerdings nicht von selbst, sondern ist von der rechtlichen Ausgestaltung des Eigentumsrechts selbst und von weiteren Rahmenbedingungen abhängig. Das Grundgesetz verpflichtet deshalb den Gesetzgeber zu einer gesellschaftsdienlichen Ausgestaltung des privaten Eigentums (,,sein Gebrauch soll zugleich dem Wohl der Allgemeinheit dienen“, Art. 14 Abs. 1 Satz 2 i.V.m. Art. 14 Abs. 2 Satz 1 GG), und, vor allem mit dem Sozialstaatsprinzip, zur Schaffung von Rahmenbedingun-

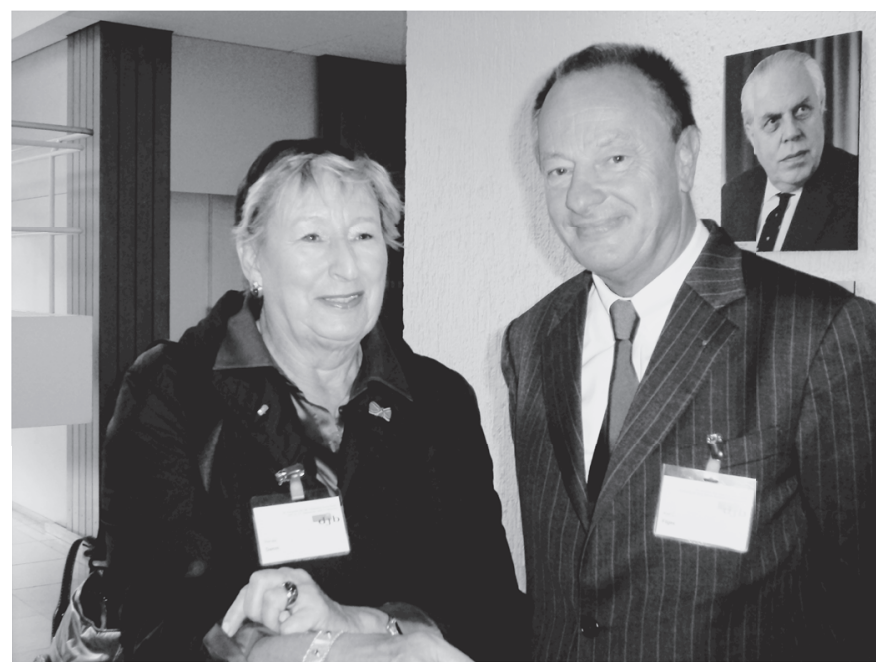

A Rechtsanwältin und Ehrenpräsidentin des djb Renate Damm mit Rechtsanwalt Axel C. Filges, Präsident der Bundesrechtsanwaltskammer (Foto: AG).

gen, die der Tendenz eines ungebändigten Wettbewerbs zur Verschärfung sozialer Gegensätze entgegenwirken.

Dass es hier Grenzen der Justiziabilität und Probleme der praktischen Umsetzung gibt - auf ein Problem der Umsetzung komme ich noch zurück - ist bekannt. Auch hier liegt aber jedenfalls die integrative Zielrichtung auf der Hand. Ebenso offensichtlich ist die integrative Bedeutung der Koalitionsfreiheit als einer wesentlichen historischen Voraussetzung für die Entschärfung von Klassengegensätzen.

Die Grundrechte, die die Freiheit der Teilnahme am Wirtschaftsleben gewährleisten, und die zugehörigen, auf Chancengleichheit und sozialen Ausgleich gerichteten Verfassungsaufträge haben integrative Bedeutung auch dadurch, dass sie Aufstiegsmobiliät möglich machen. Dasselbe gilt für das Recht auf gleichen Zugang zu öffentlichen Ämtern.

Vor allem für die USA ist die - dort besonders ausgeprägte - Aufstiegsmobiliät oft als ein wesentlicher Integrationsfaktor identifiziert worden. Man denkt dabei vor allem an die auch angesichts großer sozialer Gegensätze befriedende Wirkung der Möglichkeit, vom Tellerwäscher zum Millionär zu werden. Aufstiegsmobilität wirkt als Integrationsfaktor aber nicht nur, weil schon eine solche bloße Möglichkeit Menschen Hoffnung machen und als ein Stück Gerechtigkeit in der sozialen Ordnung empfunden werden kann. Zu dieser Empfindung neigen wir in Kontinentaleuropa ohnehin weniger als in den USA. Aufstiegsmobilität in allen Bereichen ist von besonders zentraler Bedeutung für ein Land, das große Gruppen von Migranten zu integrieren hat. Denn sie ermöglicht es solchen Gruppen, an der differenzierten Struktur der Gesellschaft teilzuhaben. Es gibt nichts, was besser geeignet wäre, die mögliche Sprengkraft von ethnischen und religiösen Guppenidentitäten zu entschärfen.

\section{Diskriminierungsverbote und gesellschaftliche Spaltungslinien}

Einen speziellen integrativen Sinn hat es auch, dass das Grundgesetz, aber auch völkerrechtliche Konventionen die beson- 


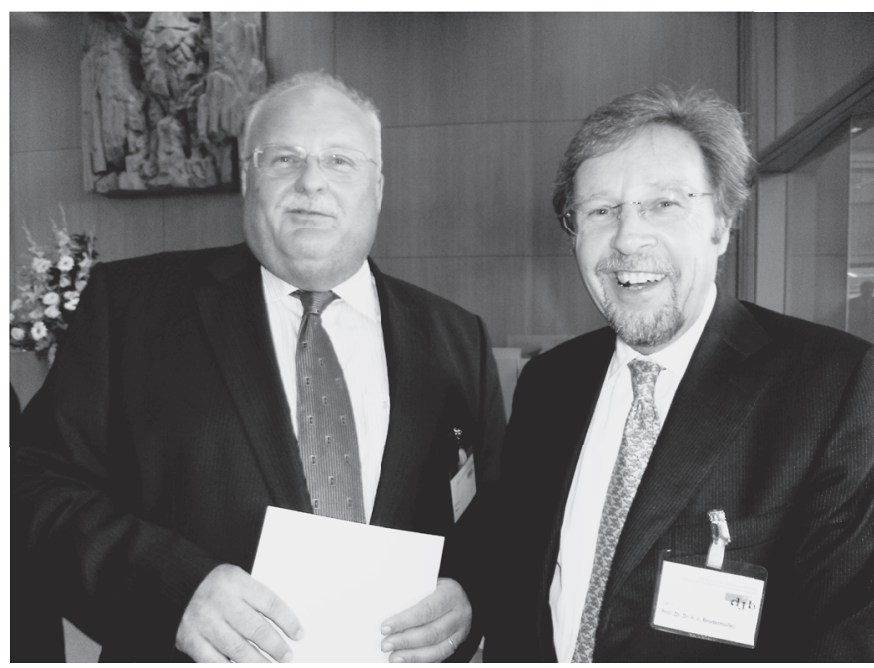

$\Delta$ Oberstaatsanwalt und Vorsitzender des Deutschen Richterbundes Christoph Frank (links) mit dem Vorsitzenden des Deutschen Familiengerichtstages Prof. Dr. Dr. h.c. Gerd Brudermüller (Foto: AG).

ders spaltungsträchtigen gesellschaftlichen Unterschiede, die Differenzen, die nach historischer Erfahrung leicht zum Anknüpfungspunkt von Polarisierungen werden, entlang denen Gesellschaften im Extremfall der Desintegration auseinanderfallen können in Bürgerkriegsparteien oder in Verfolger und Verfolgte, zum Gegenstand spezieller, besonders strikter Benachteiligungsverbote machen: Rasse, Religion, ethnische $\mathrm{Zu}-$ gehörigkeit, soziale Herkunft, politische Überzeugung. Das spezielle Verbot der geschlechtsspezifischen Diskriminierung bildet prima facie einen Sonderfall, denn an der Geschlechtergrenze können Gesellschaften nicht in derselben Weise vollkommen „auseinanderfallen“, wie dies an den Grenzen zwischen Rassen, Klassen, Ethnien oder Religionen möglich ist.

Wo verlaufen heute in unserer Gesellschaft, in Deutschland, Unterscheidungslinien, die sich in kritischer Weise vertiefen könnten?

Vielen wird da in erster Linie die Religionsverschiedenheit zwischen Christen und Muslimen einfallen. Ich halte das für zu kurz gedacht. Man muss auf die Entwicklungstendenzen sehen. Eine gruppenbildende Unterscheidungslinie, die in unserer Gesellschaft heute zu sehen ist, ist die zwischen Globalisierungsgewinnern und Globalisierungsverlierern. Sie hat die deutlichste Tendenz zur Vertiefung. Von hier aus können dann allerdings auch ethnische und religiöse Differenzen an Brisanz zunehmen - dann nämlich, wenn diese Differenzen sich mit dem ökonomischen Auseinanderfall assoziieren in der Weise, dass bestimmte ethnische und religiöse Gruppen sich perspektivlos - ohne dass gruppenbezogene Verbesserungsperspektiven sichtbar werden - auf der ökonomischen Verliererseite konzentrieren.

Die Assoziation, der Gleichlauf gesellschaftlicher Spaltungslinien verschärft Spaltungen, während umgekehrt die Überschneidung solcher Spaltungslinien - die Politikwissenschaft spricht von „cross-cutting cleavages“ - in einer Demokratie tendenziell entschärfend wirkt, also den gesellschaft- lichen Zusammenhalt stärkt, denn sie relativiert die Bedeutung der einzelnen Gruppenzugehörigkeiten und erleichtert die Akzeptanz von Mehrheitsentscheidungen oder ermöglicht sie überhaupt erst. Das liegt daran, dass bei sich überschneidenden Spaltungslinien multiple nichtkongruente Gruppenzugehörigkeiten entstehen, deshalb wechselnde Zusammensetzungen von Mehrheiten je nach dem gerade stärker betroffenen Interesse möglich werden und damit die integrationswidrige Konstellation vermieden wird, dass die Angehörigen einer bestimmten Gruppe sich als solche permanent mit allen ihren wichtigsten Interessen in der Minderheit befinden.

\section{Die Integrationsfunktion des demokratischen Prinzips}

Den Grundakt repräsentativdemokratischer Legitimation, die Wahl, hat das Bundesverfassungsgericht als „Vorgang der Integration politischer Kräfte“ bezeichnet. Warum? Weil es bei diesem Vorgang darum geht, sicherzustellen, dass nicht „gewichtige Anliegen im Volke von der Volksvertretung ausgeschlossen bleiben “. Damit ist die elementarste Funktion demokratischer Verfassungsordnung angesprochen: friedliche Kompromissfindung an die Stelle gewaltsamer Auseinandersetzung zu setzen durch die Bereitstellung von Verfahren der Produktion allgemeinverbindlicher Entscheidungen, die allen gesellschaftlichen Anliegen eine faire, auf die Gleichachtung aller Mitglieder des Gemeinwesens gegründete Chance auf Berücksichtigung und Verarbeitung sichern.

Gerade das Wahlrecht findet man übrigens in besonders offensichtlicher Weise auf die Integrationsfunktion des demokratischen Prozesses hin ausgestaltet. Integrationshalber greift es manchmal sogar zu gewissen Ungleichbehandlungen. So nimmt $\int 6$ Abs. 6 Satz 2 BWahlG die Listen von „Parteien nationaler Minderheiten“ von der 5\%-Hürde aus. Darin steckt die Überzeugung, dass eine nationale Minderheit, deren politische Repräsentation an dieser Hürde scheitern würde, integrationspolitisch betrachtet andere und größere Probleme aufwirft als eine Minderheit gleicher Größe, deren Gruppenidentität sich in einer gemeinsamen Parteipräferenz erschöpft.

Interessant ist auch die sogenannte Grundmandatsklausel des Bundeswahlgesetzes, also die Regel, wonach auch Parteien, die drei Direktmandate errungen haben, unabhängig davon in den Bundestag einziehen, ob sie die 5\%-Schwelle überwunden haben ( $\$ 6$ Abs. 6 Satz 1 BWahlG). Örtlich konzentriertes Protestpotential wird damit, ganz realistisch, ernster genommen als überregional verstreutes, und seine Einbindung in die friedenstiftenden Mechanismen demokratischer politischer Integration dementsprechend für vordringlich gehalten gegenüber dem Ziel, parteipolitischer Zersplitterung des Parlaments entgegenzuwirken.

Es gibt Verhältnisse, unter denen die Integration unterschiedlicher Bevölkerungsteile nur unter der Voraussetzung gewisser Abweichungen von strikter Gleichheit und Mehrheitsherrschaft nach dem Prinzip „one man - one vote“ funktioniert, oder jedenfalls die Verfassung des Gemeinwesens dies unterstellt und entsprechende Abweichungen vorsieht. Föderationen weisen typischerweise eine solche Abweichung auf, 
indem sie keinen gleichheitsgerechten Einfluss der Bürger der Teilstaaten auf die Gesetzgebung im Gesamtstaat vorsehen, sondern die Bürger der kleineren Teilstaaten begünstigen. Auch in unitarisch organisierten Gemeinwesen kann es inhaltliche Sonderrechte für Minderheiten geben oder besondere prozedurale Vorkehrungen, die verhindern sollen, dass eine Bevölkerungsgruppe systematisch die andere majorisiert. Im Libanon zum Beispiel bestimmt die Verfassung, dass Christen und Muslime zu gleichen Teilen im Parlament vertreten sind, obwohl dies nicht dem Anteil beider Gruppen an der libanesischen Bevölkerung entspricht. Solche verfassungsrechtlichen Besonderheiten können als die Bedingungen aufgefasst werden, unter denen unterschiedliche Bevölkerungsgruppen bereit waren, sich überhaupt unter ein gemeinsames Regiment zu begeben (oder die Gemeinsamkeit des bestehenden aufrechtzuerhalten). In diesem Sinne können unter besonderen Verhältnissen gewisse gruppenbezogene Modifikationen der strikten personenbezogenen Gleichheit integrationsdienlich und sogar Bedingung gelingender Integration sein.

Die Politikwissenschaft hat für Demokratien, in denen das Regieren nach dem Mehrheitsprinzip kraft Verfassungstext oder Verfassungspraxis weitergehenden als den üblichen Einschränkungen unterliegt, weil eine besonders ausgeprägte Fragmentierung der Gesellschaft in Teilgruppen nichts anderes zulässt, den Begriff der „consociational democracy“ (Lijphart) geprägt.

Demokratische Institutionen funktionieren, das darf man nicht unter den Tisch kehren, nicht immer in idealer Weise, und wo Dysfunktionen auftreten, da leidet ihre Integrationskraft. Sie haben aber immerhin den Vorzug, dass sie offen sind für friedliche Korrektur ihrer eigenen Dysfunktionen - nicht zuletzt auf dem Weg der Verfassungsänderung. Gegen die systembedingte Neigung demokratischer Politik zum Geldausgeben auf Kosten künftiger Generationen zum Beispiel, die Gräben zwischen den Generationen aufzureißen droht, kann man verfassungsrechtliche Grenzen für die Staatsverschuldung setzen. Auch gegen verschiedene Schwächen repräsentativdemokratischer Rückkoppelung, die vielen Demokratien zu schaffen machen - Parteioligarchisierung, übermäßiger Einfluss einzelner Interessengruppen, Machtverschiebungen zulasten der Parlamente, Defizite an Verantwortungsklarheit - lassen sich verfassungsrechtliche Kräuter anbauen.

\section{Integrationsfunktion der Verfassung und Globalisierung}

Die möglichen Integrationsleistungen der Verfassung, des durch die Verfassung institutionalisierten politischen Systems, enden allerdings da, wo die Gestaltungsmacht des jeweiligen Gemeinwesens überhaupt endet; die Integrationsleistungen der Staatsverfassung also da, wo die Gestaltungsmacht des Staates endet.

Das macht die schon erwähnte Tendenz zur Spaltung der Gesellschaft in Globalisierungsgewinner und Globalisierungsverlierer zu einem besonders schwer zu bewältigenden Integrationsproblem. Die globalisierungsbedingte Schwächung der Gestaltungsfähigkeit des Staates betrifft ja nicht zuletzt das verfassungsbasierte Integrationskonzept der Sozialstaatlichkeit. Auch hier drängt sich aber, was die Rolle der Verfassung und des Verfassungsrechts angeht, zumindest eines auf: Globalisierung ist kein Naturereignis, sondern ein durch und durch von Menschen gestalteter Prozess, gestaltet durch internationale Politik und internationale Verträge. Auch Globalisierungsfolgenbewältigung ist großenteils nur international möglich. Wie auf diesem schon komplexitätshalber schwer durchschaubaren Feld die Integrationsmechanismen des demokratischen Prozesses zur Geltung kommen, inwieweit hier gestaltungswirksame parlamentarische Mitwirkung und damit auch öffentliche Diskussion und Verantwortungszurechnung stattfindet oder die Parlamente nur praktisch vollendete Tatsachen abzusegnen haben, das sind - nicht nur, aber auch - Fragen der Verfassung und des Verfassungsrechts.

\section{Integrative Funktion der Verfassungsgerichtsbarkeit}

Über Integration durch Verfassung kann man nicht sprechen, ohne die Verfassungsgerichtsbarkeit zu erwähnen.

Das Bundesverfassungsgericht trägt zu den Integrationsleistungen der Verfassung nicht nur dadurch bei, dass es die Verfassung schützt und ihre Wirksamkeit sichert. Es hat auch wie keine andere Institution das öffentliche Bewusstsein von der Bedeutung und Verbindlichkeit der Verfassung, sowohl in allen staatlichen Institutionen als auch in der Öffentlichkeit, geprägt.

Beides hängt ganz wesentlich an der Institution der Individualverfassungsbeschwerde in unserer hiesigen Ausgestaltung. Dass jeder Bürger sich, ohne große Hürden, gegen jede mögliche Grundrechtsverletzung - auch durch alle anderen Gerichte - an das Bundesverfassungsgericht wenden und dort sein Recht finden kann, ist die wesentliche Grundlage des Vertrauens, das das Gericht und die Verfassung, nach der es Recht spricht, bei den Bürgern genießen. Damit ist es zugleich die wesentliche Grundlage der Autorität des Gerichts und - das ist daran ja das eigentlich Wichtige - der Autorität der Verfassung selbst.

Die Institution der Verfassungsbeschwerde wirkt als Mittel der Infusion von Verfassungsbewusstsein und Wertschätzung der Verfassung in alle Bereiche von Staat und Gesellschaft. Sie macht uns entsetzlich viel Arbeit. Aber dafür lohnt es sich, zu arbeiten.

Dafür, dass es dem Bundesverfassungsgericht im Wesentlichen gelungen ist, mit seiner Rechtsprechung verfassungsstabilisierend und integrativ zu wirken, sind allerdings auch noch andere Faktoren mit verantwortlich:

- Die vorgeschriebene Wahl der Richter mit Zweidrittelmehrheit.

- Die Tradition, dass das Nominationsrecht für die Richter nicht für die Dauer der Wahlperiode bei den jeweiligen Wahlsiegern liegt, sondern dass die Vorschlagsrechte zwischen den großen politischen Richtungen kontinuierlich gleichmäßig aufgeteilt sind.

- Und, mit diesen beiden Punkten eng zusammenhängend: Die auf eine gemeinsame Entscheidung gerichtete Kultur sehr intensiver Beratung bei den Senatsentscheidungen. 
All das begünstigt eine „mittige“ Rechtsprechung, die mehr integriert als polarisiert.

\section{Demokratievertrauen als Integrationsfaktor}

Das Integrationspotential unserer Verfassung ist kein Selbstläufer. Auch unter einer solchen Verfassung kann integrationspolitisch Vieles falsch gemacht werden - erst recht, wenn es um die Integration von Menschen geht, die nicht Bürger und also auch nicht Wähler sind und in Bezug auf die deshalb die Integrationsmechanismen der freiheitlichen Demokratie nur eingeschränkt zum Tragen kommen.

$\mathrm{Zu}$ den fehlerträchtigen Dispositionen gehört mangelndes Vertrauen in die Integrationskraft unserer freiheitlichen Demokratie. Daraus entsteht Angst, Reizbarkeit, leichte Provozierbarkeit und die Neigung zu symbolischen Gefechten anstelle geduldiger Arbeit an den realen Problemen, die sich stellen. All das ist Gift für die Integration unterschiedlicher Gruppen in einer Gesellschaft.

Warum dieses mangelnde Vertrauen? Die freiheitliche Demokratie als ein labiles Gebilde, der Erosion der Werte preis- gegeben, schwach in der Selbstbehauptung - diese Sichtweise hat starke Wurzeln in der europäischen und ganz besonders in der deutschen Kulturgeschichte. Sie hängt nicht nur mit der schon erwähnten Homogenitätslehre zusammen, sondern auch damit, dass die Demokratie, diese unmartialische Veranstaltung, als eine weibische, und damit eo ipso auch schwächliche, Angelegenheit verachtet war. Eine Assoziation, die gerade dieser Versammlung sicher besonders fernliegt, von der Weltbühne aber noch lange nicht abgetreten ist.

Die Deutschen haben ihre historische Demokratieverachtung in den Jahren seit dem Krieg überwunden. Das notwendige Vertrauen in die Stärke und Überzeugungskraft von Freiheit und Demokratie fehlt uns manchmal noch. Dieses Vertrauen ist keins, mit dem man sich zurücklehnen könnte. Die demokratische Verfassungsordnung ist keine Schlaraffenordnung, sondern die Ordnung, die es jedem erlaubt, an ihrer Verbesserung zu arbeiten, und die mehr als jede andere darauf ausgerichtet ist, aus Fehlern zu lernen. Ich wünsche Ihnen für Ihre Tagung die Arbeitsfreude und die Zuversicht, die man daraus beziehen kann.

\section{Familiennachzug und Spracherwerb: Allgemeine Grundsätze und Rechtsprechung}

\author{
Univ.-Prof. Dr. Margarete Schuler-Harms, Vorsitzende der Kom- \\ mission Recht der sozialen Sicherung, Familienlastenausgleich \\ im djb, Helmut-Schmidt-Universität, Hamburg
}

\section{Der Familiennachzug in Zahlen}

Im Aufenthaltsgrund des Familiennachzugs gehen Geschlecht und Familienzugehörigkeit eine besonders enge Verbindung ein. Einige Daten und Fakten sollen die große Bedeutung des Familiennachzugs im Rahmen der Migration und den hohen Anteil der Frauen im Familiennachzug verdeutlichen.

Der Familiennachzug bildete $2007^{1}$ den häufigsten Grund für die Zuwanderung nach Deutschland und in die Europäische Union. Nach Deutschland zogen rund 55.000 von rund 400.000 Ausländern aus familiären Gründen. Eine besondere Gruppe bilden dabei vor allem die sogenannten Drittstaatsangehörigen, also Ausländer ohne Staatsangehörigkeit in einem EU-Mitgliedstaat. Rund 29 Prozent der einreisenden Drittstaatsangehörigen zogen 2007 zu Ehepartnern oder anderen Familienangehörigen nach. Den Hauptanteil bildeten dabei Türkinnen und Türken, von denen nahezu die Hälfte zum Zweck des Familiennachzugs einreiste. ${ }^{2}$ Kein anderer Aufenthaltsgrund wird von Drittstaatsangehörigen annähernd häufig geltend gemacht. Knapp 20 Prozent der Drittstaatsangehörigen kommen, um eine Schulbildung, eine Ausbildung, ein Studium oder einen Sprachkurs zu absolvieren, ca. 15,3 Prozent bezwecken die Aufnahme einer Beschäftigung und etwa 2,6 Prozent reisen aus humanitären Gründen ein. ${ }^{3}$ Die Zuwanderung aus familiären Gründen unterscheidet sich von anderen Zuwanderungsgründen auch dadurch, dass der Aufenthalt in der Bundesrepublik regelmäßig langfristig oder gar auf Dauer angelegt ist.

Der Anteil der Frauen im Familiennachzug übersteigt den der Männer deutlich. ${ }^{4}$ Der Gesamtanteil der Frauen an den Zuzügen ins Bundesgebiet liegt seit etwa 15 Jahren konstant bei 40 bis 43 Prozent, der Anteil der Migrantinnen beim Zuzug zu Beschäftigungszwecken bei etwa einem Drittel. Demgegenüber wurden von den insgesamt ca. 55.000 aus familiären Gründen erteilten Aufenthaltstiteln über die Hälfte (knapp 52 Prozent, im Vorjahr 55 Prozent) an nachziehende Ehefrauen erteilt. Ein knappes Viertel (22,3 Prozent) ging an nachziehende Ehemänner und etwa ebenso viel (22,4 Prozent) an nachziehende Kinder. Der Anteil sonstiger Familienangehöriger lag bei 3,6 Prozent und umfasste ganz überwiegend den

1 Vgl. Bundesamt für Ausländer und Flüchtlinge (BAMF), Migrationsbericht 2007, $30 \mathrm{ff} .$, abrufbar unter www.bmi.bund.de.

2 Vgl. BAMF, Migrationsbericht 2007, 120 (Übersichten). Der Anteil der Türkinnen und Türken am Ehegatten- und Familiennachzug insgesamt betrug 33,4\% im Jahr 1997 und immerhin noch 21,9\% im Jahr 2007, vgl. BAMF, Migrationsbericht 2007, 121.

3 BAMF, Migrationsbericht 2007, Übersicht 32.

4 BAMF, Migrationsbericht 2007, $30 \mathrm{f}$. 